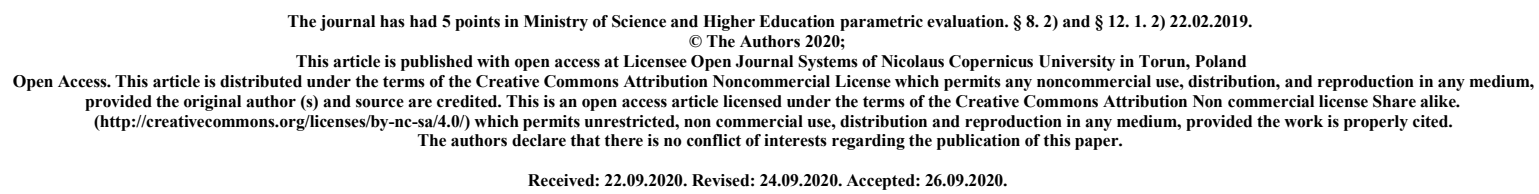

\title{
Secondary and side effects of particular Selective Serotonin Reuptake Inhibitors (SSRIs) antidepressants - literature review
}

\section{Ewa Krzewicka-Romaniuk ${ }^{1}$, Dagna Siedlecka ${ }^{1}$, Artur Romaniuk ${ }^{2}$}

${ }^{1}$ Department of Pathophysiology, Medical University of Lublin, Lublin, Poland

${ }^{2}$ Praktyka Lekarza Rodzinnego „Familia” ul. Niepodległości 29 21-040 Świdnik

Key words: SSRI; antidepressants; side effects

\begin{abstract}
Serotonin reuptake inhibitors (SSRIs), along with serotonin norepinephrine reuptake inhibitors (SNRIs) and norepinephrine dopamine reuptake inhibitors (NDRIs), are the first-line drugs in the treatment of depression. Pharmacotherapy of psychiatric disorders with SSRIs is widely practised. Although SSRIs are thought to be relatively safe group of drugs, it is worth to remember that particular SSRIs agents are related to unique secondary and side effects. Knowledge about basic differences between each medication in this group is essential to choose suitable treatment for every patient.
\end{abstract}




\section{INTRODUCTION:}

Serotonin reuptake inhibitors (SSRIs), along with serotonin norepinephrine reuptake inhibitors (SNRIs) and norepinephrine dopamine reuptake inhibitors (NDRIs), are the first-line drugs in the treatment of depression.(1) Pharmacotherapy of different psychiatric disorders with SSRIs is widely practised. In the United States, one in eight people have used SSRIs in the past 10 years.(2)

The basic mechanism of action of SSRIs is the inhibition of serotonin reuptake. The drug molecule attaches to the serotonin transporter, which prevents the uptake of 5hydroxytryptamine, what results in the increase of the amount of the neurotransmitter in the synapse. There are 6 drugs in the SSRI group. Each of them, in addition to its primary effect of serotonin reuptake, has separate secondary mechanisms of action and side effects.(1)

Although SSRIs are better tolerated than most other types of antidepressants,(3) some patients may experience serious and arduous side effects like cardiotoxicity, anticholinergic effects, sedation or sexual dysfunctions, including Post SSRIs Sexual Dysfunction (PSSD). In our review we want to go briefly through selected secondary and side effects of particular SSRIs.

\section{FLUOXETINE}

Fluoxetine is an antagonist of 5-HT2C receptors, which results in the release of noradrenaline (NA) and dopamine (DA). Under normal circumstances, serotonin inhibits NA and DA release through 5-HT2c receptors. Blocking the 5HT2C receptors leads to a disinhibition of NA and DA release, therefore 5HT2C receptor antagonists such as fluoxetine are called NA and DA dysinhibitors (NDDI -noradrenaline and dopamine dysinihibitors). The action on 5HT2C receptors may explain the activating properties of the drug. Fluoxetine also inhibits the cytochrome CYP450 2D6 and 3A4. Through this action, fluoxetine may increase the levels of other drugs. Additionaly, it is a drug with a particulary long half-life. Doctors should stay cautious while using fluoxetine in children. Patients should be carefully monitored for the induction of mania or suicidal ideation. Children may $\begin{array}{llll}\text { experience } & \text { a } & \text { slowdown } & \text { growth.(1) }\end{array}$ 
While SSRIs are typically viewed as weight-neutral, there have been reports of weight gain with fluoxetine treatment compared to placebo.(4)

Fluoxetine may affect cardiovascular system. Mild bradycardia has been observed during chronic treatment with fluoxetine. Moreover, there are increasing number of case reports on dysrhythmia, syncope and orthostatic hypotension associated with fluoxetine and another SSRIs treatment and overdose.(5)

\section{SERTRALINE}

The uniqueness of sertraline is that it binds to sigma-1 receptors and the dopamine transporter (DAT). Therefore in addition to inhibiting serotonin reuptake, it is also a dopamine reuptake inhibitor (DRI). Although the importance of an action on sigma-1 receptors is not well understood, it is believed that it may contribute to the anxiolytic effect of sertraline and may be useful in the treatment of psychotic depression.(1)

Sertraline is most likely of the SSRIs to cause gastrointestinal disturbances for example diarrhea(6). Other common side effects of sertraline are: decreased sexual desire or ability and failure to discharge semen (in men).(7)

\section{PAROXETINE}

Paroxetine is often the drug of choice in patients suffering from depression and accompanying anxiety symptoms. Its effectiveness may be related to its anticholinergic activity - antagonism of M1 muscarinic receptors. The mild anticholinergic effect can accelerate the onset of the therapeutic effect but may also induce mild anticholinergic side effects. What is more paroxetine may increase the anticholinergic effects of other drugs.

In addition to inhibiting serotonin reuptake, paroxetine has a slight inhibitory effect on the norepinephrine transporter and is a strong inhibitor of CYP450 2D6, which may increase the concentration of other drugs in the blood. Paroxetine is both a substrate and an inhibitor of this cytochrome, which may lead to a rapid drop in drug concentration upon discontinuation and related withdrawal symptoms. Withdrawal symptoms are more likely to occure while using paroxetine than with other SSRIs.(1) 
A common side effect of paroxetine is an excessive sedation. Paroxetine is thought to be the most anticholinergic and most sedating of all SSRIs.(6) Inhibition of nitric oxide synthase (NOS) may be responsible for sexual dysfunction with paroxetine use. Unlike most SSRIs, paroxetine is a Category D risk for use in pregnancy.(1) Mild bradycardia has been observed during chronic treatment with paroxetine.(5)

\section{FLUVOXAMINE}

Fluvoxamine has a potentially important secondary mechanism of action involving actions at the sigma-1 receptors. Fluvoxamine is believed to be an antagonist of these receptors, contributing to its anxiolytic efficacy. The effect of fluvoxamine on sigma-1 receptors is greater than that of sertraline. Fluvoxamine is a strong inhibitor of CYP450 $1 \mathrm{~A} 2$ and 3A4, which may increase the blood levels of various drugs. A common side effect of fluvoxamine is excessive sedation.(1) Mild bradycardia has been observed during chronic treatment with fluvoxamine. (5)

\section{CITALOPRAM:}

Citalopram consists of two enantiomers, $\mathrm{R}$ and $\mathrm{S}$. Their combination is racemic citalopram, which has a mild antihistaminergic and a CYP 450 2D6 inhibitory effect related to the R-enantiomer. Activating the R-enantiomer on SERT may interfere with the inhibitory effect of the S-enantiomer on this transporter. This interaction may weaken the inhibition of SERT, decrease the concentration of serotonin in the synapsia and thus decrease the therapeutic efficacy of citalopram.(1)

Citalopram is thought to be more cardiotoxic than other SSRIs. (6) In some studies citalopram-treatment was associated with reduced heart rate. This reduction occurred within the first week of the treatment without further reduction later. In a small group of citalopram-treated patients $(3-4 \%)$ with normal heart rate at baseline bradycardia was developed. Furthermore, citalopram treatment was associated with a non-specific, insignificant prolongation of QT interval irrespective of age. In younger group of the patients a statistically significant decrease in T-wave amplitude was also demonstrated.(5)

\section{ESCITALOPRAM}

Escitalopram is essentially R-enantiomer-free citalopram. Thus, its antidepressant effect is only associated with the pure inhibition of SERT by the S-enantiomer. Removal of the R-enantiomer renders escitalopram devoid of antihistaminergic and 
CYP4502D6 inhibitory effects. Furthermore, due to the removal of the R-enantiomer which interferes with SERT inhibition, it is possible to use lower doses of the drug. Escitalopram is one of the best-tolerated SSRIs with the fewest adverse events associated with CYP450.(1)

SUMMARY:

Although SSRIs are thought to be relatively safe group of drugs, it is worth to remember that particular SSRIs agents are related to unique secondary and side effects. Knowledge about basic differences between each medication in this group is essential to choose suitable treatment for every patient.

\section{REFERENCES}

1. Stephen M. Stahl. Psychofarmakologia w ilustracjach LEKI PRZECIWDEPRESYJNE. Gdańsk: VIA MEDICA; 2010.

2. Bala A, Nguyen HMT, Hellstrom WJG. Post-SSRI Sexual Dysfunction: A Literature Review. Sexual Medicine Reviews. 1 styczeń 2018;6(1):29-34.

3. Selective serotonin reuptake inhibitors (SSRIs) - Side effects [Internet]. nhs.uk. 2017 [cytowane 21 wrzesień 2020]. Dostępne na: https://www.nhs.uk/conditions/ssriantidepressants/side-effects/

4. (PDF) The Pharmacologic Management of SSRI-Induced Side Effects: A Survey of Psychiatrists [Internet]. ResearchGate. Dostępne na: https://www.researchgate.net/publication/10900637_The_Pharmacologic_Management_o f_SSRI-Induced_Side_Effects_A_Survey_of_Psychiatrists

5. Pacher P, Kecskemeti V. Cardiovascular Side Effects of New Antidepressants and Antipsychotics: New Drugs, old Concerns? Current pharmaceutical design. 2004;10(20):2463.

6. Barbara Fadem. Behavioral Science. SIXTH EDITION. Wolters Kluwer Lippincott Williams \& Wilkins; 2014. 
7. Sertraline (Oral Route) Side Effects - Mayo Clinic [Internet]. [cytowane 21 wrzesień 2020]. Dostępne na: https://www.mayoclinic.org/drugs-supplements/sertraline-oralroute/side-effects/drg-20065940 